\title{
Low Dose Imaging by STEM Ptychography Using Pixelated STEM Detector
}

Ryusuke Sagawa ${ }^{1}$, Hiroki Hashiguchi ${ }^{1}$, Thomas Isabell ${ }^{2}$, Robert Ritz ${ }^{3}$, Martin Simson ${ }^{3}$, Martin Huth $^{3}$, Heike Soltau ${ }^{3}$, Gerardo T. Martinez ${ }^{4}$, Peter D. Nellist ${ }^{4}$ and Yukihito Kondo ${ }^{1}$

1. EM Business Unit, JEOL Ltd., Tokyo, Japan.

2. JEOL USA Inc., Boston, USA.

3. Department of Materials, University of Oxford, Oxford, UK.

4. PNDetector GmbH, München, Germany.

In material and biological studies, low dose imaging to see an intact sample before electron damage is requested, since we have become to observe beam sensitive materials frequently as we expand the extent of objects for observation. The low dose imaging is requested in scanning transmission electron microscopy (STEM) observation as well, as the method is quite useful in combination with analytical data obtained by energy dispersive X-ray spectroscopy (EDS) and/or electron energy loss spectroscopy (EELS). In conventional STEM imaging, we normally use single channel detectors with a fixed-shaped scintillator, which simply sums up the electron signal on the detector plane. Due to this integration, rich specimen information in convergent beam electron diffraction (CBED) pattern which is projected on a scintillator plane is averaged and partly lost. For efficient extraction of the sample information, a segmented multichannel STEM detector has been used to perform a differential phase contrast (DPC) imaging, where difference of signals between the opposing areas on the detector is used, resulting in a successful visualization of electromagnetic field or the phase difference in a specimen [1,2]. Meanwhile, direct electron detectors with fast frame rate of several thousand frames per second and several ten thousands pixels have recently been commercialized and used as pixelated STEM detectors, which records CBED patterns for each STEM probe position. [3,4]. With the obtained 4-dimensional (4D) dataset, any type of STEM images can be synthesized as one can freely define an integration area on CBED patterns. On the other hand, with this pixelated STEM detector, we have a chance to apply the detector for low dose imaging, since the extracted phase information from the $4 \mathrm{D}$ dataset is very rich and giving an image of high signal-to-noise $(\mathrm{S} / \mathrm{N})$ ratio.

We have developed a pixelated STEM detector (4DCanvas ${ }^{\mathrm{TM}}$, JEOL, ) with a fast direct electron CCD image sensor (pnCCD, PNDetector) whose maximum readout speed is $4,000 \mathrm{fps}[3,4]$. The confirmed operable accelerating voltage is $20 \sim 300 \mathrm{kV}$. For experiments in this paper, the detector was integrated into an aberration corrected electron microscope (NEOARM, JEOL). As this detector is installed below the conventional ADF detector, we can simultaneously record a 4D dataset and an ADF-STEM image.

The ptychography, which reconstructs a phase image of an object, is one of the applications using this pixelated STEM detector. The method is effectively used for obtaining a high S/N STEM image [5,6]: the integration areas (shape and position) are dynamically changed for a corresponding spatial frequency. The Figure 1 (a) and (b) show phase maps obtained from a monolayer graphene sample. Only those areas that have an intensity variation as the probe scans (i.e., the overlap areas between the BF and the diffracted disks that contribute to the phase contrast) are colored with blue and red for each spatial frequency. One then selects the areas and synthesizes a high $\mathrm{S} / \mathrm{N}$ image without loss of phase contrast of the sample. This precise and complicate manipulation is difficult to perform by the conventional single channel detectors. Figure 2 shows a comparison between (a) an ADF STEM image and (b) a ptychographically reconstructed phase image of a single-walled carbon nanotube (SWCNT) recorded simultaneously. The phase image gives apparently higher image contrast and $\mathrm{S} / \mathrm{N}$ ratio. In the FFT 
patterns, the higher frequency signals are observed in the phase image than in the ADF. This is because the ptychography method effectively extracts the sample information inside the BF disk which intrinsically has more signal than in the area of ADF. Moreover, using ptychography, we can correct residual aberration in the reconstructed image, because once we have obtained the phase map, we can access and manipulate the phase of the electron wave [7]. This will also help low dose observation because one cannot adjust defocus or two-fold astigmatism aberration precisely before recording. Thus, the electron dose required for such alignment is minimized.

\section{References:}

[1] N Shibata et al, J. Electron Microsc., 59 (2010), p. 473.

[2] N Shibata et al, Nature Physics, 8 (2012), p. 611.

[3] H Ryll et al, J. Instrum., 11 (2016), p. 04006.

[4] R Sagawa et al, Microsc. Microanal., 23 (2017), p. 52.

[5] PD Nellist, B.C. McCallum and J.M. Rodenburg, Nature, 374 (1995), p. 630.

[6] TJ Pennycook et al, Ultramicroscopy, 151 (2015), p. 160.

[7] H Yang et al, Nature Communications, 7 (2016), p. 12532.
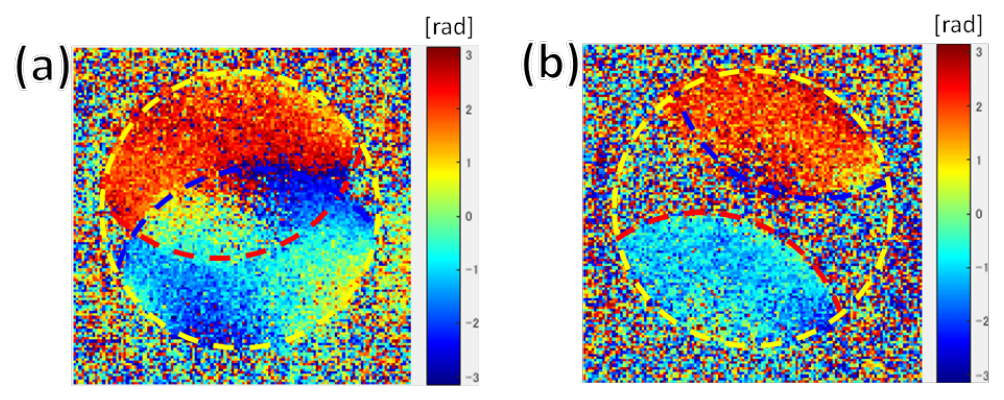

Figure 1. Phase maps of CBED patterns for the spatial frequencies corresponding to (a) $\left(\begin{array}{llll}0 & -1 & 1 & 0\end{array}\right)$ and (b) $\left(\begin{array}{llll}1 & -2 & 1 & 0\end{array}\right)$ reflections of graphene. The BF disks are indicated by the yellow dashed circles and the diffracted disks the blue and the red ones.
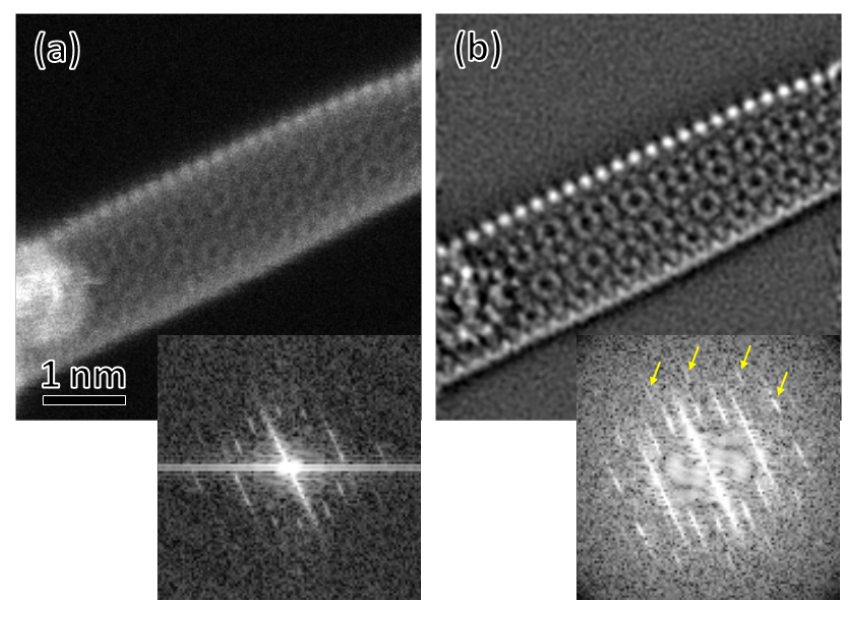

Figure 2. Comparison between (a) an ADF STEM image and (b) a ptychographically reconstructed phase image from a single walled carbon nanotube. The yellow arrows in the FFT pattern of the phase image show higher spatial frequencies that are invisible in the ADF image. The probe current was approximately $7 \mathrm{pA}$ and the frame rate of the 4DCanvas ${ }^{\mathrm{TM}}$ was 4,000 fps (corresponds to $250 \mu$ s of dwell time of scanning probe). 